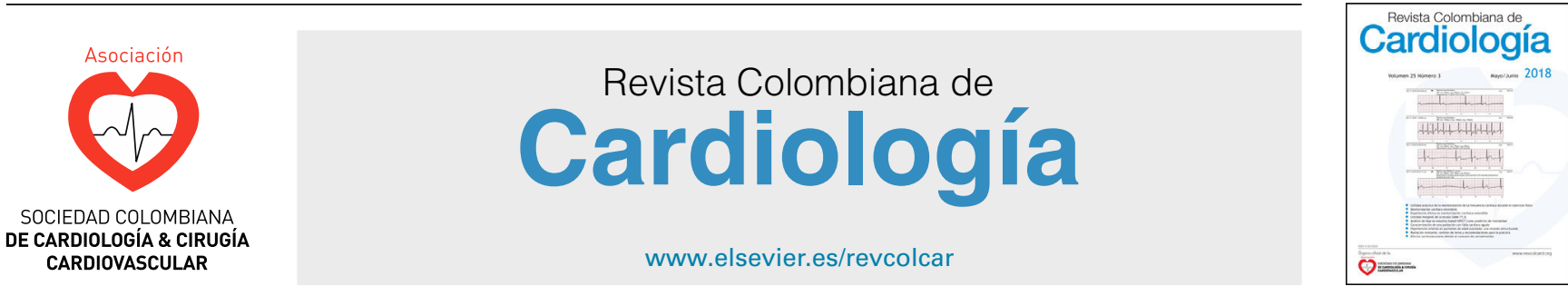

CARDIOLOGÍA DEL ADULTO - ARTÍCULO ORIGINAL

\title{
Caracterización de la enfermedad cardiaca en pacientes embarazadas y desenlaces hospitalarios materno-fetales
}

\author{
Edison Muñoz-Ortiz ${ }^{\mathrm{a}, \mathrm{b}, *}$, Jairo A. Gándara-Ricardo a,b, \\ Jesús A. Velásquez-Penagos ${ }^{\mathrm{a}, \mathrm{c}}$, Natalia Giraldo-Ardila ${ }^{\mathrm{d}}$, Ana M. Betancur-Pizarro ${ }^{\mathrm{a}}$, \\ Edwin F. Arévalo-Guerrero ${ }^{a}$, Fernando M. Fortich-Hoyos ${ }^{a}$ y Juan M. Sénior-Sánchez ${ }^{a, b}$
}

a Clínica de Embarazo y Enfermedad Cardiaca, Unidad Funcional Cardiopulmonar y Vascular Periférico, Hospital Universitario San Vicente Fundación, Medellín, Colombia

b Sección de Cardiología, Departamento de Medicina Interna, Universidad de Antioquia, Medellín, Colombia

c Sección de Perinatología y Alto Riesgo Obstétrico, Departamento de Obstetricia y Ginecología, Universidad de Antioquia, Medellín, Colombia

' Unidad Neonatal, Hospital General de Medellín, Medellín, Colombia

Recibido el 10 de mayo de 2018; aceptado el 16 de diciembre de 2019

Disponible en Internet el 23 de octubre de 2020

\section{PALABRAS CLAVE \\ Embarazo; \\ Cardiopatía; \\ Complicaciones \\ maternas; \\ Complicaciones \\ neonatales}

\begin{abstract}
Resumen
Introducción: la enfermedad cardiaca durante el embarazo puede llevar a deterioro clínico e incluso a la muerte de la madre o el feto. En nuestro medio hay pocos datos al respecto.

Materiales y métodos: estudio observacional, analítico, de cohorte retrospectiva, de gestantes con enfermedad cardiaca, en el que se incluyeron pacientes con cardiopatía congénita o adquirida, o arritmias, que requirieran hospitalización o intervención urgente por manifestación de síntomas. Se excluyeron pacientes con insuficiencias valvulares leves o moderadas, o estenosis leves, y pacientes sin estudio ecocardiográfico o sin información del parto. Se determinaron eventos cardiacos primarios y secundarios, así como eventos obstétricos y neonatales.

Resultados: se incluyeron 104 pacientes con igual número de embarazos. La evaluación por Cardiología fue en promedio a las 32 semanas. La fracción de expulsión promedio fue del $61 \%$ y el 23,1\% tenía dilatación del ventrículo derecho. El 26,9\% de las pacientes tenían alguna intervención cardiovascular previa.

Las cardiopatías congénitas fueron el diagnóstico más frecuente $(51,9 \%)$, seguido por enfermedad valvular $(25,9 \%)$, arritmias $(15,4 \%)$ y disfunción ventricular izquierda $(4,8 \%)$. Los eventos cardiacos primarios se presentaron en $13,5 \%$ de las pacientes, en tanto que los secundarios en el 14,4\%. La cesárea por indicación cardiaca fue sólo en el $21,2 \%$. Los eventos neonatales ocurrieron en el $36,5 \%$; y los eventos obstétricos en el $14,4 \%$.
\end{abstract}

\footnotetext{
* Autor para correspondencia.

Correo electrónico: edison.munoz@sanvicentefundacion.com (E. Muñoz-Ortiz).
} 
Conclusiones: pese al alto riesgo y las etiologías complejas, las embarazadas con enfermedad cardiaca reciben evaluación tardía por cardiología, lo que puede explicar las altas tasas de eventos cardiacos maternos y neonatales. Se requieren grupos con experiencia para el manejo de estas pacientes.

(C) 2020 Sociedad Colombiana de Cardiología y Cirugía Cardiovascular. Publicado por Elsevier España, S.L.U. Este es un artículo Open Access bajo la licencia CC BY-NC-ND (http:// creativecommons.org/licenses/by-nc-nd/4.0/).

\section{KEYWORDS \\ Pregnancy; \\ Heart disease; \\ Maternal \\ complications; \\ Newborn \\ complications}

Characterisation of heart disease in pregnant patients and mother-newborn hospital outcomes

\begin{abstract}
Introduction: Heart disease during pregnancy can lead to a clinical deterioration, and even to the death of the mother or the child. There is lack of data as regards this in the scientific literature.

Material and methods: A retrospective, observational, and analytical study was conducted on a cohort of pregnant women with heart disease. The study included patients with congenital or acquired heart disease or arrhythmias that required hospital admission or urgent treatment due to onset of symptoms. Patients with mild or moderate valve insufficiency, as well as those with no ultrasound data or information about their delivery, were excluded. Primary and secondary cardiac events, as well as obstetric and neonatal events were recorded.

Results: The study included 104 patients with an equal number of pregnancies. The evaluation by Cardiology was at 32 weeks on average. The mean ejection fraction was $61 \%$, and $23.1 \%$ had enlargement of the right ventricle. More than one-quarter $(26.9 \%)$ of the patients had some previous cardiovascular treatment.

Congenital heart disease was the most common diagnosis $(51.9 \%)$, followed by valve disease (25.9\%), arrhythmias (15.4\%), and left ventricular dysfunction (4.8\%). Primary cardiac events were observed in $13.5 \%$ of the patients, whilst $14.4 \%$ had secondary events. Caesarean section due to a cardiac indication was only performed in $21.2 \%$. Neonatal events were recorded in $36.5 \%$, with obstetric events in $14.4 \%$.

Conclusions: Despite the high risk and aetiological complexity, pregnant women with heart disease were assessed at a late stage by cardiology. This may explain the high rates of maternal and neonatal events. Groups with experience are required to manage these patients.

(c) 2020 Sociedad Colombiana de Cardiología y Cirugía Cardiovascular. Published by Elsevier España, S.L.U. This is an open access article under the CC BY-NC-ND license (http:// creativecommons.org/licenses/by-nc-nd/4.0/).
\end{abstract}

\section{Introducción}

La enfermedad cardiaca se manifiesta en el 0,2 al $4 \%$ de las mujeres en gestación en los países industrializados occidentales $^{1}$, cifra de gran impacto si se tiene en cuenta que el embarazo y el periodo periparto se asocian con múltiples cambios circulatorios que pueden llevar al deterioro clínico y hasta a un 10 a $15 \%$ de las causas de mortalidad materna, aunado a un aumento de morbimortalidad fetal ${ }^{2,3}$.

En nuestro medio hay pocos datos sobre la epidemiología de la enfermedad cardiaca y los resultados materno-fetales hospitalarios, lo cual es necesario para poder tomar medidas en salud pública en busca de mejorar los resultados del binomio madre-hijo; por eso en este trabajo se busca caracterizar la enfermedad cardiaca en las pacientes gestantes en un centro de referencia y definir desenlaces hospitalarios materno-fetales.

\section{Materiales y métodos}

Estudio observacional, analítico, de cohorte retrospectiva, con una muestra no probabilística por conveniencia. La muestra consistió en 104 pacientes embarazadas que ingresaron al servicio de gineco-obstetricia de una institución de alta complejidad, entre el $1 .^{\circ}$ de enero de 2010 y el 31 de diciembre de 2015, incluyendo pacientes con cardiopatías congénitas o adquiridas, corregidas o no, y con arritmias que requirieran manejo hospitalario. Se revisaron las historias clínicas de la madre y del neonato, y se diligenció un formulario para la recolección de la información en el programa informático Microsoft Excel; posteriormente, se hizo el análisis de los datos con STATA 13. Los desenlaces cardiovasculares, neonatales y obstétricos fueron corroborados por un cardiólogo, una neonatóloga y un gineco-obstetra, respectivamente. Se excluyeron pacientes con enfermedad valvular leve o moderada según los criterios de las guías de enfermedad valvular de la Asociación Americana del Corazón y el Colegio Americano de Cardiología de $2014^{4}$ y aquellas que no tenían estudio ecocardiográfico o sin información del parto luego de al menos cuatro intentos de contacto telefónico.

Las variables tomadas de la historia clínica incluyeron datos demográficos, como edad, seguridad social, estado de paridad, edad gestacional al momento de la primera evaluación por Cardiología y al momento del parto; hábitos, 
como tabaquismo y alcohol, y comorbilidades de acuerdo con las definiciones de las guías vigentes, como hipertensión arterial, diabetes mellitus, dislipidemia y otras que implicaran tomar un tratamiento crónico; antecedentes cardiovasculares, como intervenciones cardiovasculares previas al embarazo, quirúrgicas o percutáneas, enfermedad o complicación cardiaca previa al embarazo, saturación de oxígeno al momento del ingreso, cianosis, medicamentos de uso crónico; con énfasis en anticoagulantes respecto a su indicación, tipo y seguimiento. En cuanto a los hallazgos ecocardiográficos se recolectó la fracción de expulsión, la presencia o no de dilatación del ventrículo derecho y el tipo de cardiopatía encontrada. Se verificó la vía del parto, y en caso de parto por cesárea se revisó si la indicación fue obstétrica o cardiaca. La indicación de cesárea por enfermedad cardiaca se definió acorde con las recomendaciones de las guías de embarazo y enfermedad cardiaca de la Sociedad Europea de Cardiología ${ }^{1}$.

Los desenlaces hospitalarios materno-fetales y obstétricos se catalogaron de la siguiente manera:

- Eventos cardiacos primarios: edema pulmonar, taquiarritmias o bradiarritmias sintomáticas sostenidas que necesitaran tratamiento, accidente cerebrovascular, paro cardiaco o muerte cardiaca.

- Eventos cardiacos secundarios: deterioro en dos o más clases de la NYHA (New York Heart Association) comparado con el basal, necesidad de procedimiento invasivo cardiaco urgente en el embarazo o posparto intrahospitalario.

- Eventos neonatales: parto prematuro definido como menor a 37 semanas de gestación, peso bajo para la edad gestacional cuando era menor al percentil 10, síndrome de dificultad respiratoria, hemorragia intraventricular, muerte fetal definida como aquella mayor o igual a 20 semanas de gestación, o muerte neonatal cuando se presentaba desde el momento del nacimiento hasta los 28 días posparto. Adicional a los eventos neonatales se indagó por la presencia de aborto, definido como la pérdida de la gestación menor a 20 semanas.

- Eventos obstétricos: muerte materna no cardiaca, trastorno hipertensivo asociado al embarazo y hemorragia posparto, definida como una pérdida sanguínea mayor a $500 \mathrm{ml}$ en parto vaginal o mayor de $1.000 \mathrm{ml}$ en cesárea.

Las variables categóricas se resumen en valores absolutos y relativos. Para las variables continuas se realizó el supuesto de normalidad con la prueba de Shapiro-Wilk. Las variables con distribución normal se resumen con promedios y desviación estándar y las variables que no cumplieron el supuesto de normalidad con medianas y rango intercuartil (RIQ).

\section{Resultados}

Entre el $1 .^{\circ}$ de enero de 2010 y el 31 de diciembre de 2015 se incluyeron 104 pacientes con igual número de embarazos. De 2 pacientes no se tuvo ecocardiografía porque se consideró realizarla de forma ambulatoria y no se conoció posteriormente el resultado por pérdida del seguimiento, y hubo 3 pacientes adicionales sin información del parto.
Tabla 1 Características generales de las pacientes

\begin{tabular}{|c|c|}
\hline Variable & $\mathrm{n}=104$ \\
\hline Edad en años, mediana (rango) & $22(15-40)$ \\
\hline $\begin{array}{l}\text { Número de embarazos previos, mediana } \\
\text { (rango) }\end{array}$ & $2(0-7)$ \\
\hline $\begin{array}{l}\text { Edad gestacional al momento del parto en } \\
\text { semanas, mediana (rango) }\end{array}$ & $34(6-40)$ \\
\hline $\begin{array}{l}\text { Edad gestacional en la primera evaluación } \\
\text { por Cardiología en semanas, mediana } \\
\text { (rango) }\end{array}$ & $\begin{array}{l}32(5- \\
\text { posparto) }\end{array}$ \\
\hline $\begin{array}{l}\text { Porcentaje de saturación de oxígeno al } \\
\text { ingreso (rango) }\end{array}$ & $\begin{array}{l}94,7(50- \\
100)\end{array}$ \\
\hline \multicolumn{2}{|c|}{ Régimen de afiliación en salud - número de pacientes (\%) } \\
\hline Contributivo & $23(22,1)$ \\
\hline Subsidiado & $70(67,3)$ \\
\hline Sin afiliación & $11(11,6)$ \\
\hline \multicolumn{2}{|l|}{$\begin{array}{l}\text { Antecedente de tabaquismo - número de } \\
\text { pacientes (\%) }\end{array}$} \\
\hline Nunca & $99(95,2)$ \\
\hline Extabaquismo & $4(3,9)$ \\
\hline Consumo activo & $1(0,9)$ \\
\hline \multicolumn{2}{|l|}{$\begin{array}{l}\text { Consumo de alcohol en la gestación - } \\
\text { número de pacientes (\%) }\end{array}$} \\
\hline No & $102(98,1)$ \\
\hline Sí & $2(1,9)$ \\
\hline \multicolumn{2}{|l|}{$\begin{array}{l}\text { Estado funcional clasificado al ingreso - } \\
\text { número de pacientes (\%) }\end{array}$} \\
\hline NYHA I & $77-(74)$ \\
\hline NYHA II & $17-(16,4)$ \\
\hline NYHA III & $7-(6,7)$ \\
\hline NYHA IV & $3-(2,9)$ \\
\hline \multicolumn{2}{|l|}{$\begin{array}{l}\text { Riesgo OMS modificado - número de } \\
\text { pacientes (\%) }\end{array}$} \\
\hline I & $12(11,5)$ \\
\hline II & $59(56,7)$ \\
\hline III & $16(15,4)$ \\
\hline IV & $17(16,4)$ \\
\hline \multicolumn{2}{|l|}{ Hallazgos ecocardiográficos } \\
\hline Fracción de expulsión en \% (rango) & $61(20-85)$ \\
\hline \multicolumn{2}{|l|}{$\begin{array}{l}\text { Dilatación del ventrículo derecho - número } \\
\text { de pacientes (\%) }\end{array}$} \\
\hline No & $80(76,9)$ \\
\hline Sí & $24(23,1)$ \\
\hline \multicolumn{2}{|l|}{ Comorbilidades - número de pacientes (\%) } \\
\hline Hipertensión arterial & $12(11,6)$ \\
\hline Hipotiroidismo & $4(3,8)$ \\
\hline Hipertiroidismo & $1(0,9)$ \\
\hline Diabetes mellitus tipo 2 & $2(1,9)$ \\
\hline Enfermedad cerebrovascular isquémica & $2(1,9)$ \\
\hline Embolia pulmonar & $1(0,9)$ \\
\hline Lupus eritematoso sistémico & $2(1,9)$ \\
\hline Arteritis de Takayasu & $1(0,9)$ \\
\hline Enfermedad mixta del tejido conectivo & $1(0,9)$ \\
\hline
\end{tabular}


Tabla 1 (Continuación)

\begin{tabular}{|c|c|}
\hline Variable & $\mathrm{n}=104$ \\
\hline Síndrome antifosfolípido & $1(0,9)$ \\
\hline Púrpura trombocitopénica inmune & $1(0,9)$ \\
\hline Epilepsia & $1(0,9)$ \\
\hline Infección por VIH & $1(0,9)$ \\
\hline \multicolumn{2}{|l|}{$\begin{array}{l}\text { Medicamentos de uso crónico - número de } \\
\text { pacientes (\%) }\end{array}$} \\
\hline Ninguno & $75(72,1)$ \\
\hline Calcioantagonistas & $5(4,8)$ \\
\hline Alfa-metildopa & $5(4,8)$ \\
\hline Furosemida & $5(4,8)$ \\
\hline IECA & $5(4,8)$ \\
\hline ASA & $5(4,8)$ \\
\hline Betabloqueador & $4(3,9)$ \\
\hline Levotiroxina & $4(3,9)$ \\
\hline Enoxaparina & $4(3,9)$ \\
\hline Esteroide & $3(2,9)$ \\
\hline Warfarina & $3(2,9)$ \\
\hline ARA-II & $1(0,9)$ \\
\hline Espironolactona & $1(0,9)$ \\
\hline Cloroquina & $1(0,9)$ \\
\hline Azatioprina & $1(0,9)$ \\
\hline Sildenafil & $1(0,9)$ \\
\hline Hidroclorotiazida & $1(0,9)$ \\
\hline Metformina & $1(0,9)$ \\
\hline Clonidina & $1(0,9)$ \\
\hline Digoxina & $1(0,9)$ \\
\hline \multicolumn{2}{|l|}{$\begin{array}{l}\text { Indicación de anticoagulación - número de } \\
\text { pacientes (\%) }\end{array}$} \\
\hline Ninguna & $98(94,2)$ \\
\hline Válvula cardiaca mecánica & $4(3,9)$ \\
\hline Tromboembolia venosa & $1(0,9)$ \\
\hline Trombofilia & $1(0,9)$ \\
\hline \multicolumn{2}{|l|}{$\begin{array}{l}\text { Tipo de anticoagulación - número de } \\
\text { pacientes (\%) }\end{array}$} \\
\hline Ninguno & $98(94,3)$ \\
\hline Warfarina & $2(1,9)$ \\
\hline Enoxaparina sin medir niveles de anti-Xa & $3(2,9)$ \\
\hline $\begin{array}{l}\text { Warfarina seguida por enoxaparina sin } \\
\text { medir niveles de anti-Xa }\end{array}$ & $1(0,9)$ \\
\hline
\end{tabular}

ARA-II: antagonista del receptor de angiotensina II, ASA: ácido acetilsalicílico, IECA: inhibidor de la enzima convertidora de angiotensina, NYHA: New York Heart Association, OMS: Organización Mundial de la Salud, VIH: virus de inmunodeficiencia humana.

Las características generales de las pacientes se muestran en la tabla 1. La edad gestacional promedio en que se dio la primera evaluación por Cardiología fue a las 32 semanas (rango 5 a 40 semanas), 2 de las pacientes se evaluaron en el periodo posparto. La fracción de expulsión promedio fue del $61 \%$, y el $23,1 \%$ tenían dilatación del ventrículo derecho. Tres pacientes recibieron anticoagulación con warfarina durante la gestación con dosis menores o iguales a $5 \mathrm{mg}$ al día en todos los casos.

Entre los eventos cardiacos previos al embarazo que motivaron la atención, se encontró que el 5,8\% habían tenido clínica de falla cardiaca, el 3,9\% episodios de taquiarritmias
Tabla 2 Tipo de intervenciones cardiovasculares previas al embarazo

\begin{tabular}{|c|c|}
\hline Variable & $n=104$ \\
\hline $\begin{array}{l}\text { Corrección de aneurisma de Valsalva - número de } \\
\text { pacientes (\%) }\end{array}$ & $1(0,9)$ \\
\hline Ablación de arritmia - número de pacientes (\%) & $2(1,9)$ \\
\hline Cirugía valvular - número de pacientes (\%) & $7(6,7)$ \\
\hline $\begin{array}{l}\text { Cirugía valvular + corrección de comunicación } \\
\text { interventricular + marcapasos - número de } \\
\text { pacientes }(\%)\end{array}$ & $1(0,9)$ \\
\hline $\begin{array}{l}\text { Implante de marcapasos o cardiodesfibrilador - } \\
\text { número de pacientes (\%) }\end{array}$ & $2(1,9)$ \\
\hline $\begin{array}{l}\text { Corrección de comunicación interauricular e } \\
\text { interventricular - número de pacientes (\%) }\end{array}$ & $1(0,9)$ \\
\hline $\begin{array}{l}\text { Corrección de aneurisma de aorta - número de } \\
\text { pacientes (\%) }\end{array}$ & $1(0,9)$ \\
\hline $\begin{array}{l}\text { Cierre quirúrgico de ductus arterioso persistente - } \\
\text { número de pacientes (\%) }\end{array}$ & $2(1,9)$ \\
\hline $\begin{array}{l}\text { Corrección de tetralogía de Fallot - número de } \\
\text { pacientes (\%) }\end{array}$ & $1(0,9)$ \\
\hline $\begin{array}{l}\text { Corrección endovascular de coartación de aorta - } \\
\text { número de pacientes (\%) }\end{array}$ & $2(1,9)$ \\
\hline $\begin{array}{l}\text { Corrección quirúrgica de coartación de aorta - } \\
\text { número de pacientes (\%) }\end{array}$ & $1(0,9)$ \\
\hline $\begin{array}{l}\text { Corrección quirúrgica de comunicación } \\
\text { interventricular - número de pacientes (\%) }\end{array}$ & $3(2,9)$ \\
\hline $\begin{array}{l}\text { Corrección quirúrgica de comunicación } \\
\text { interauricular - número de pacientes (\%) }\end{array}$ & $3(2,9)$ \\
\hline $\begin{array}{l}\text { Corrección de canal aurículo-ventricular - número } \\
\text { de pacientes }(\%)\end{array}$ & $1(0,9)$ \\
\hline
\end{tabular}

y el 1,9\% bloqueo aurículo-ventricular avanzado que requirió implante de marcapasos. Además, el $26,9 \%$ de las pacientes habían tenido algún tipo de intervención cardiovascular previo al embarazo (tabla 2).

Las cardiopatías congénitas fueron el diagnóstico primario más frecuente en las gestantes evaluadas, con el $51,9 \%$, seguido por la enfermedad cardiaca valvular en el $25,9 \%$, arritmias en el $15,4 \%$ y la disfunción ventricular izquierda en el 4,8\%. Otras alteraciones cardiacas menos frecuentes fueron la hipertensión arterial pulmonar idiopática y los tumores cardiacos, con $0,9 \%$ cada uno (fig. 1). Los diagnós-

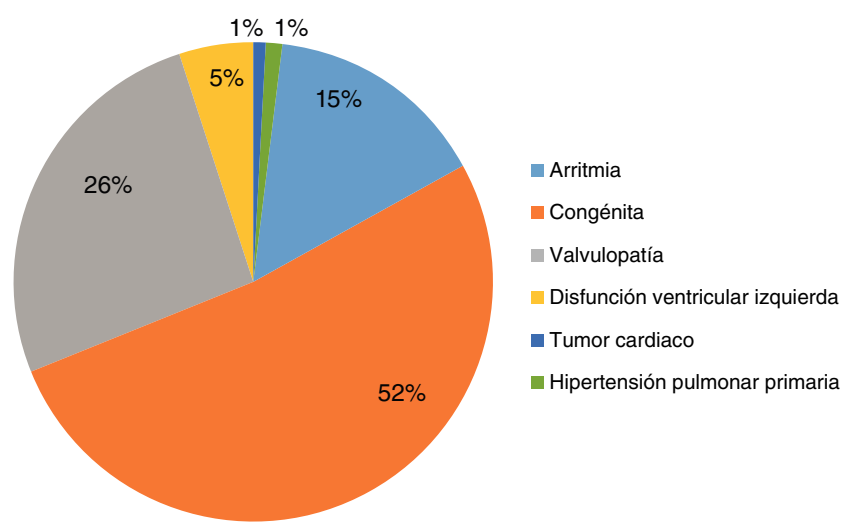

Figura 1 Tipos de cardiopatías encontradas como diagnóstico principal. 
Tabla 3 Diagnósticos cardiacos específicos

\begin{tabular}{|c|c|}
\hline Variables & $\mathrm{n}=104$ \\
\hline Insuficiencia mitral - número de pacientes (\%) & $11(10,6)$ \\
\hline Ductus arterioso persistente & $10(9,6)$ \\
\hline Comunicación interventricular & $10(9,6)$ \\
\hline $\begin{array}{l}\text { Comunicación interventricular con síndrome de } \\
\text { Eisenmenger }\end{array}$ & $2(1,9)$ \\
\hline $\begin{array}{l}\text { Comunicación interventricular + comunicación } \\
\text { interauricular }\end{array}$ & $4(3,9)$ \\
\hline $\begin{array}{l}\text { Comunicación interventricular con síndrome de } \\
\text { Eisenmenger + tronco arterioso común }\end{array}$ & $1(0,9)$ \\
\hline Comunicación interauricular & $8(7,7)$ \\
\hline $\begin{array}{l}\text { Comunicación interauricular + drenaje venoso } \\
\text { anómalo }\end{array}$ & $1(0,9)$ \\
\hline $\begin{array}{l}\text { Taquicardia supraventricular por fenómeno de } \\
\text { reentrada }\end{array}$ & $8(7,7)$ \\
\hline Aorta bivalva sin estenosis o insuficiencia grave & $6(5,8)$ \\
\hline Disfunción ventricular izquierda & $5(4,8)$ \\
\hline Doble lesión mitral & $5(4,8)$ \\
\hline Válvula mecánica & $3(2,9)$ \\
\hline Anomalía de Ebstein & $3(2,9)$ \\
\hline Automatismo ventricular aumentado & $3(2,9)$ \\
\hline Coartación de aorta corregida & $3(2,9)$ \\
\hline $\begin{array}{l}\text { Bloqueo aurículo-ventricular avanzado sin } \\
\text { marcapasos previo }\end{array}$ & $2(1,9)$ \\
\hline Estenosis mitral & $2(1,9)$ \\
\hline Estenosis pulmonar congénita & $2(1,9)$ \\
\hline Estenosis aórtica & $2(1,9)$ \\
\hline Insuficiencia mitral + insuficiencia aórtica & $1(0,9)$ \\
\hline $\begin{array}{l}\text { Transposición congénitamente corregida de } \\
\text { grandes arterias + válvula } \\
\text { mecánica + marcapasos }\end{array}$ & $1(0,9)$ \\
\hline Endocarditis infecciosa & $1(0,9)$ \\
\hline Válvula biológica & $1(0,9)$ \\
\hline $\begin{array}{l}\text { Presencia de marcapasos por bloqueo } \\
\text { aurículo-ventricular completo }\end{array}$ & $1(0,9)$ \\
\hline Insuficiencia aórtica & $1(0,9)$ \\
\hline Tetralogía de Fallot corregida & $1(0,9)$ \\
\hline Hipertensión arterial pulmonar primaria & $1(0,9)$ \\
\hline Taquicardia atrial & $1(0,9)$ \\
\hline Tetralogía de Fallot no corregida & $1(0,9)$ \\
\hline Fibrilación auricular & $1(0,9)$ \\
\hline Canal aurículo-ventricular corregido & $1(0,9)$ \\
\hline Fibroelastoma & $1(0,9)$ \\
\hline
\end{tabular}

ticos cardiacos específicos y su frecuencia de aparición se describen en la tabla 3.

\section{Eventos cardiacos}

Los eventos cardiacos primarios se presentaron en el $13,4 \%$ de las pacientes (tabla 4), entre los que hubo una muerte materna cardiaca por hipertensión arterial pulmonar idiopática. Los eventos cardiacos secundarios se presentaron en el $14,4 \%$, dados por 14 pacientes que presentaron deterioro en su clase funcional mayor a 2 clases de la NYHA, 2 de esas pacientes con deterioro funcional requirieron intervención cardiaca urgente y una paciente más requirió intervención cardiovascular urgente.
Tabla 4 Eventos cardiacos primarios en las pacientes con desenlaces

\begin{tabular}{lc}
\hline Evento & $\mathrm{n}=104$ \\
\hline Edema pulmonar - número de pacientes (\%) & $9(8,7)$ \\
$\begin{array}{l}\text { Taquiarritmia sostenida que requirió tratamiento - } \\
\text { número de pacientes (\%) }\end{array}$ & $3(2,9)$ \\
$\begin{array}{l}\text { Bradicardia que necesitó tratamiento - número de } \\
\text { pacientes (\%) }\end{array}$ & $1(0,9)$ \\
$\begin{array}{l}\text { Muerte cardiaca - número de pacientes (\%) } \\
\text { Múm }\end{array}$ & $1(0,9)$ \\
\hline
\end{tabular}

Tabla 5 Eventos neonatales

\begin{tabular}{ll}
\hline Evento & $\mathrm{n}=104$ \\
\hline $\begin{array}{l}\text { Parto prematuro - número de pacientes (\%) } \\
\text { Parto prematuro + bajo peso para la edad }\end{array}$ & $16(15,3)$ \\
$\quad$ gestacional - número de pacientes $(\%)$ & $5(4,8)$ \\
$\begin{array}{l}\text { Parto prematuro + síndrome de dificultad } \\
\quad \text { respiratoria - número de pacientes }(\%)\end{array}$ & $4(3,9)$ \\
$\begin{array}{l}\text { Parto prematuro + bajo peso para la edad } \\
\quad \text { gestacional + síndrome de dificultad respiratoria }\end{array}$ & $2(1,9)$ \\
$\quad$ - número de pacientes (\%) & $2(1,9)$ \\
$\begin{array}{l}\text { Bajo peso para la edad gestacional } \\
\text { Muerte fetal - número de pacientes }(\%)\end{array}$ & $1(0,9)$ \\
\hline
\end{tabular}

Tabla 6 Eventos obstétricos

\begin{tabular}{ll}
\hline Evento & $\mathrm{n}=104$ \\
\hline Trastorno hipertensivo asociado al embarazo & $8(7,7)$ \\
Hemorragia posparto & $5(4,8)$ \\
Trastorno hipertensivo asociado al & $1(1,9)$ \\
$\quad$ embarazo + hemorragia posparto & \\
\hline
\end{tabular}

\section{Eventos neonatales y obstétricos}

Siete pacientes presentaron aborto espontáneo (6,7\%), 45 pacientes tuvieron parto vaginal $(43,3 \%)$ y 52 fueron llevadas a cesárea (50\%). La indicación cardiaca para realizar cesárea fue para 11 pacientes $(21,2 \%)$.

Los eventos neonatales se presentaron en el 28,9\% (tabla $5)$. Hubo una muerte fetal relacionada con múltiples malformaciones no warfarínicas.

Por su lado, los eventos obstétricos se presentaron en el $14,4 \%$; los más frecuentes fueron los trastornos hipertensivos en el $9,6 \%$ y la hemorragia posparto en el $1,9 \%$. No hubo muertes maternas de causa obstétrica (tabla 6).

\section{Discusión}

La evaluación de la embarazada con cardiopatía ha sido un tema poco abordado en nuestro medio. Este es el primer estudio en Colombia que busca una caracterización clínica más amplia, en la que se evalúan desenlaces maternos y fetales hospitalarios, en gestantes con enfermedad cardiaca confirmada. Múnera et al. ${ }^{5}$, hicieron un gran aporte con la descripción de las frecuencias de enfermedad cardiaca en una cohorte de 153 embarazadas con sospecha de enfermedad cardiaca, pero en su estudio buscaban determinar 
la utilidad diagnóstica y pronóstica de la ecocardiografía; además, sólo el 52\% tuvo hallazgos anormales, lo cual no descarta necesariamente una cardiopatía, ya que algunas arritmias de importancia clínica aparecen en corazones estructuralmente normales. Esto es relevante puesto que esta es la principal causa de muerte materna por enfermedad cardiovascular en el Reino Unido ${ }^{3}$. Nuestros datos son los primeros de un programa multidisciplinario estructurado que se enfoca en esta población específica, con trascendencia en la salud pública, ya que en Colombia el $39 \%$ de las muertes maternas se deben a causas indirectas, es decir, a condiciones preexistentes conocidas o no, que se agravan o se descubren en la gestación o el postparto, en cuyo caso el $3.7 \%$ de estas muertes corresponde a cardiopatías ${ }^{6}$. Pese a que las gestantes son un grupo prioritario de atención en salud, en este trabajo queda evidente que la evaluación por cardiología en las embarazadas con cardiopatía es muy tardía (en promedio a las 32 semanas), lo cual también explica, al menos en parte, la alta tasa de desenlaces cardiovasculares primarios y secundarios que se encontraron, tan graves como una muerte materna o el hallazgo elevado de edema pulmonar $(8,7 \%)$, cuando en series muy grandes se muestra que es una complicación infrecuente del embarazo $0^{7,8}$, si bien en esos registros no se enfocaron específicamente en pacientes con enfermedad cardiaca. Se evidencia una alta proporción de desenlaces neonatales $(29,8 \%)$, que es levemente más alta que el $27,8 \%$ reportado por Khairy et al. ${ }^{9}$, aunque inferior a los desenlaces neonatales reportados por Múnera et al. en los que la sola tasa de prematuridad fue del $34 \%$, además de $32 \%$ de peso bajo para la edad gestacional, $5 \%$ de muertes fetales y 2,5\% de muertes neonatales. Estos resultados neonatales adversos implican altos costos en la atención del neonato, aunado a los riesgos de morbilidad, mortalidad y discapacidad a largo plazo de estos niños. Consideramos que estos desenlaces elevados se pueden explicar por la falta de identificación temprana de la enfermedad desde la etapa preconcepcional o etapas tempranas del embarazo, de inadecuado y tardío seguimiento y de carencia de medidas terapéuticas tempranas. En nuestra experiencia, desde la conformación del programa de clínica de embarazo y enfermedad cardiaca, se ha podido confirmar que aquellas pacientes que tienen un inicio de seguimiento temprano y estrecho han tenido excelentes resultados materno-fetales (datos aún no publicados).

Es llamativo que a pesar de que el $89 \%$ de las pacientes pertenecen a un régimen de afiliación al sistema de salud, no se hubiera garantizado el acceso temprano a servicios de salud de alta complejidad, lo que indica falta de gestión de políticas públicas, como lo es la atención de la paciente gestante. Por otro lado, se trata de mujeres muy jóvenes, con 2 hijos en promedio, por lo que postulamos que las condiciones socioculturales son relevantes para garantizar un seguimiento adecuado y un tratamiento temprano.

En cuanto a los tipos de cardiopatía, la enfermedad cardiaca congénita fue la más encontrada, lo cual concuerda con datos reportados en el mundo, como en el registro europeo de enfermedad cardiaca y embarazo ${ }^{10}$ (ROPAC por su sigla en inglés), al cual nuestro programa aportó pacientes, y lo reportado por Múnera et al. ${ }^{5}$ Esto se convierte en un reto, pues muchas de las cardiopatías encontradas implican riesgos que generalmente son muy altos, y de ahí la pre- mura de insistir en la necesidad de grupos con experiencia en el manejo de estas pacientes. Además, la enfermedad cardiaca valvular, que fue la segunda cardiopatía en frecuencia, requiere afrontar retos adicionales, como el riesgo de endocarditis y, muy especialmente, en las pacientes con válvula mecánica, y el uso adecuado de la anticoagulación en un estado protrombótico como lo es el embarazo y con los riesgos materno-fetales que implican las diferentes opciones de tratamiento. Las arritmias y la falla cardiaca, que fueron las otras alteraciones frecuentes, tienen sus propios retos de manejo debido a los riesgos fetales inherentes a las terapias disponibles para estas condiciones, así como a los requerimientos de equipos altamente especializados para poder atender a las pacientes. Un estudio de una unidad cardio-obstétrica de Irak encontró hallazgos con algunas variaciones, pues los autores reportaron como cardiopatía más frecuente la enfermedad valvular seguida por las cardiopatías congénitas ${ }^{11}$, pero no evaluaron los desenlaces materno-fetales, como sí se hizo en el presente estudio.

Es de resaltar que el $14 \%$ de las pacientes tuvieron, además, complicaciones propias de la gestación, como trastorno hipertensivo asociado al embarazo y hemorragia postparto, que por sí solas son las principales causas de muerte materna en nuestro medio ${ }^{6}$, lo que genera mayor impacto desfavorable en pacientes con enfermedad cardiaca previa. Por otro lado, se ha reportado mayor incidencia de hemorragia postparto en pacientes con enfermedad cardiaca congénita, no relacionada con la anticoagulación ni con la vía del parto ${ }^{12}$. Las tasas de cesárea en este tipo de pacientes reportadas en la literatura son variables (11-90\%); por sí sola esta vía de parto se asocia a mayores complicaciones, más aún si es de urgencia, incluso en las gestantes sin enfermedad cardíaca, por lo que la decisión de realizarla idealmente se debe tomar de manera conjunta y planeada ${ }^{13}$.

Reconocemos que este trabajo tiene las limitaciones propias de un estudio retrospectivo, tales como falta de algunas variables ecocardiográficas como la medición de velocidad de regurgitación tricuspídea como marcador de hipertensión pulmonar; sin embargo, esta cohorte desarrollada como preludio de la organización del Programa de cardiopatía y embarazo en un centro de alta complejidad, permite hacer un primer acercamiento a la epidemiología de las embarazadas con enfermedad cardiaca y, con trabajos futuros de carácter prospectivo que se están realizando en la actualidad, poder contar con datos adicionales que permitan ofrecer una atención óptima y obtener mejores desenlaces.

\section{Conclusiones}

Este estudio permite conocer las enfermedades cardiacas más frecuentes en la población gestante, así como los riesgos materno-fetales, los cuales fueron altos. A pesar de ser de alto riesgo y con etiologías complejas caracterizadas en el presente trabajo, las embarazadas con enfermedad cardiaca tienen evaluaciones tardías por cardiología, lo que puede explicar las altas tasas de eventos cardiacos maternos y neonatales. Estos desenlaces muestran la necesidad del manejo de estas pacientes en un programa con experiencia para poder obtener mejores resultados. 


\section{Financiación}

\section{Ninguna.}

\section{Conflicto de intereses}

Ninguno.

\section{Bibliografía}

1. Regitz-Zagrosek V, Blomstrom C, Borghi C, Cifkova R, Ferreira $R$, Foidart JM, et al. ESC Guidelines on the management of cardiovascular diseases during pregnancy The task force on the management of cardiovascular diseases during pregnancy of the European society of cardiology (ESC). Eur Heart J. 2011;32:3147-97.

2. Sliwa K, Bohm M. Incidence and prevalence of pregnancy related Heart disease. Cardiovasc Res. 2014;101:554-60.

3. Knight M, Nair M, Tuffnell D, Kenyon S, Shakespeare J, Brocklehurst P. (eds.) on behalf of MBRRACE-UK. Saving Lives. En: Improving Mothers' Care - Surveillance of maternal deaths in the UK 2012-14 and lessons learned to inform maternity care from the UK and Ireland Confidential Enquiries into Maternal Deaths and Morbidity 2009-14. Oxford: National Perinatal Epidemiology Unit, University of Oxford; 2016.

4. Nishimura RA, Otto CM, Bonow RO, Carabello BA, Erwin JP, Guyton RA, et al. 2014 AHA/ACC Guidelines for the management of patients with valvular heart disease: executive summary: a report of American College of Cardiology/American Heart Association Task Force on practice Guidelines. Circulation. 2014;129:2440-92.
5. Múnera AG, Manrique RD, Orrego JC, Mesa C, Betancur C, Gómez M. Valor pronóstico de los estudios ecocardiográficos en el diagnóstico y seguimiento de la patología cardiaca durante el embarazo. Rev Fed Arg Cardiol. 2012;41:114-20.

6. Cuevas EL. Informe del evento mortalidad materna 2012. Instituto Nacional de Salud Pública. Vigilancia y control en salud pública. Disponible en http://www.ins.gov.co:81/lineasde-accion/SubdireccionVigilancia/Informe \%20de \%20Evento \%20Epidemiolgico/MORTALIDAD \%20MATERNA \%202012.pdf.

7. Sciscione AC, Ivester T, Largoza M, Manley J, Shlossman P, Colmorgen GH. Acute pulmonary edema in pregnancy. Obstet Gynecol. 2003;101:511-5.

8. Dennis AT, Solnordal CB. Acute pulmonary oedema in pregnant women. Anaesthesia. 2012;67:646-59.

9. Khairy P, Ouyang DW, Fernandes SM, Lee-Parritz A, Economy KE, Landzberg MJ. Pregnancy outcomes in women with congenital heart disease. Circulation. 2006;113:517-24.

10. Van Hagen IM, Boersma E, Johnson MR, Thorne SA, Parsonage WA, Subias E, et al. Global cardiac risk assesment in the registry of pregnancy and cardiac disease: results of a registry from the European society of cardiology. Eur J Heart Fail. 2016;18:523-33.

11. Farhan HA, Yaseen IF. Heart disease in pregnancy -clinical pattern and prevalence: initial data from the first cardio-maternal unit in Iraq. BMC Res Notes. 2019;12:491.

12. Cauldwell M, Von Klemperer K, Uebing A, Swan L, Steer PJ, Gatzoulis $M$, et al. Why is post-partum haemorrhage more common in women with congenital heart disease? Intern J Cardiology. 2016;218:285-90.

13. Ruys TPE, Roos-Hesselink JW, Pijuan-Domènech A, Vasario E, Gaisin IR, Lung B, et al. Is a planned caesarean section in women with cardiac disease beneficial? Heart. 2015;101:530-6. 